Communications in Physics, Vol. 25, No. 1 (2015), pp. 21-28

DOI:10.15625/0868-3166/25/1/5509

\title{
TAYLOR'S SERIES AND DISPERSION RELATION ANALYSES OF THE VECTOR PION FORM FACTOR AND THEIR COMPARISON WITH PERTURBATIVE AND NON PERTURBATIVE CALCULATIONS
}

\author{
TRAN NGUYEN TRUONG \\ Centre de Physique Théorique, \\ Ecole Polytechnique \\ F91128 Palaiseau, France
}

E-mail:Tran.Truong@cpht.polytechnique.fr

Received 20 November 2014

Accepted for publication 28 November 2014

\begin{abstract}
The first three coefficients of the Taylor's series expansion of the vevtor pion form factor as a function of the momentum transfer are evaluated using the experimental data on the pion form factor and the $P$-wave $\pi \pi$ phase shifts. The real part of the form factor as a function of energy is also calculated by dispersion relation. Comparisons there results with Chiral Perturbation Theory and unitarized models are given.
\end{abstract}

Keywords: ???.

The purpose of this note is three-fold. First, a systematic procedure is given to calculate the coefficients of the Taylor's series expansion of the form factor around the origin $s=0$ in terms of experimental data. Second the real parts of the form factor for a wider range of energy are calculated using also experimental data. Third, these results are compared with those given by existing theoretical calculations in order to evaluate their reliability. It turns out that only models satisfying the elastic unitarity, giving rise to the $\rho$ resonance, are valid. Chiral Perturbation calculations at one and two-loop disagree, at very low energy, with the Taylor's series expansion and, at moderate energy, with the dispersion relation results.

Chiral Perturbation Theory (ChPT) [1-4] is a well-defined perturbative procedure allowing one to calculate systematically low energy phenomenon involving soft pions. It is now widely used to analyze the low energy pion physics not only when the interaction is not strong but also in the presence of the resonance as long as the energy region of interest is sufficiently far from the resonance. In this scheme, the unitarity relation is satisfied perturbatively order by order.

In this note we want to examine critically the ChPT approach and wish to emphasize that unless that this calculation was followed by a unitarisation procedure, its results for a number of physical processes would not be meaningful. This is so because in any perturbative scheme, it is important that the magnitude of the calculated term should be much larger than that of the same

unité propre 014 du CNRS

(C)2015 Vietnam Academy of Science and Technology 
order which is uncalculable because of its non-perturbative origin. To make this point clear, let us examine the one-loop ChPT calculation of the vector pion form factor $V(s)[3,4]$ :

$$
V^{\text {pert. }}(s)=1+\frac{s}{s_{R}}+\frac{1}{96 \pi^{2} f_{\pi}^{2}}\left(\left(s-4 m_{\pi}^{2}\right) H_{\pi \pi}(s)+\frac{2 s}{3}\right)
$$

where $f_{\pi}=0.93 \mathrm{GeV}$ and the r.m.s. radius of the vector form factor is related to $s_{R}$ by the definition $V^{\prime}(0)=\frac{1}{6} r_{V}^{2}=1 / s_{R}$. The function $H_{\pi \pi}(s)$ is given by:

$$
H_{\pi \pi}(s)=\left(2-2 \sqrt{\frac{s-4 m_{\pi}^{2}}{s}} \ln \frac{\sqrt{s}+\sqrt{s-4 m_{\pi}^{2}}}{2 m_{\pi}}\right)+i \pi \sqrt{\frac{s-4 m_{\pi}^{2}}{s}}
$$

fors $>4 m_{\pi}^{2}$; for other values of s, $H_{\pi \pi}(s)$ can be obtained by analytic continuation.

From this definition, it is clear that the third term on the r.h.s. of Eq. (1), being an analytic function with the same singularity as $V(s)$, can be expanded as a Taylor's series at $s=0$ with a leading term behaving as $s^{2}$ at small $s$. For small $s$ it is a reasonable approximation to take only the first few terms of this series.

The unitarised version of Eq. (1), obtained by the inverse amplitude, the Padé approximant or the N/D methods, is given by $[7,8]$ :

$$
V(s)=\frac{1}{1-s / s_{R}-\frac{1}{96 \pi^{2} f_{\pi}^{2}}\left\{\left(s-4 m_{\pi}^{2}\right) H_{\pi \pi}(s)+2 s / 3\right\}}
$$

It is obvious that Eq. (3) has the Breit-Wigner resonance character while that from Eq. (1) does not, although their amplitude and first derivative are identical at $s=0$. Furthermore, if the parameter $s_{R}$ was fixed by the the r.m.s. radius, the $\rho$ mass would come out to be slightly low compared measured $\rho$ mass. Neglecting this last problem at the moment, the Taylor's series expansion around $s=0$ reveals that Eq. (3) gives rise to a coefficient of the $s^{2}$ term as $\left(1 / s_{R}\right)^{2} \simeq$ $4.0 \mathrm{GeV}^{-4}$ which is much larger than that coming from the third term of Eq. (1), $1 /\left(960 \pi^{2} m_{\pi}^{2} f_{\pi}^{2}\right) \simeq$ $0.63 \mathrm{GeV}^{-4}$. This is the signal of the failure of the perturbation method. While it is difficult to detect the presence of this term using experimental data at low energy, this failure would show up at higher energy and with more accurate data.

It could be argued, however, that this discrepancy could come from the unitarisation scheme, because Eq. (3) fits the data only approximatively. It is therefore desirable to have a direct proof that this discrepancy is real and only depends on the experimental data.

Because the vector pion form factor $V(s)$ is an analytic function with a cut from $4 m_{\pi}^{2}$ to $\infty$, the $n^{\text {th }}$ subtracted dispersion relation for $V(s)$ reads:

$$
V(s)=a_{0}+a_{1} s+\ldots a_{n-1} s^{n-1}+\frac{s^{n}}{\pi} \int_{4 m_{\pi}^{2}}^{\infty} \frac{\operatorname{Im} V(z) d z}{z^{n}(z-s-i \varepsilon)}
$$

where $n \geq 0$ and, for simplicity, the series around the origin is considered. The polynomial on the R.H.S. of this equation will be referred in the following as the subtraction constants and the last term on the R.H.S. as the dispersion integral (DI). The evaluation of DI as a funtion of $s$ will be done later. Notice that $a_{n}=V^{n}(0) / n$ ! is the coefficient of the Taylor series expansion for $V(s)$, where $V^{n}(0)$ is the nth derivative of $V(s)$ evaluated at the origin. The condition for Eq. (4) to be valid was that, on the real positive s axis, the limit $s^{-n} V(s) \rightarrow 0$ as $s \rightarrow \infty$. By the Phragmen 
Lindeloff theorem, this limit would also be true in any direction in the complex s-plane and hence it is straightforward to prove Eq. (4). The coefficient $a_{n+m}$ of the Taylor's series is given by:

$$
a_{n+m}=\frac{1}{\pi} \int_{4 m_{\pi}^{2}}^{\infty} \frac{\operatorname{Im} V(z) d z}{z^{(n+m+1)}}
$$

where $m \geq 0$. The meaning of this equation is clear: under the above stated assumption, not only the coefficient $a_{n}$ can be calculated but all other coefficients $a_{n+m}$ can also be calculated.

Below the inelastic threshold, from unitarity of the S-matrix, $V(s)$ must have the phase of the P-wave elastic $\pi \pi$ phase shifts [9]. From the available experimental data, the inelastic effect will manifest only at an energy above $1.3 \mathrm{GeV} \mathrm{[10].} \mathrm{Therefore,} \mathrm{below} \mathrm{this} \mathrm{energy} \mathrm{one} \mathrm{has:}$

$$
\operatorname{Im} V(z)=|V(z)| \sin \delta(z)
$$

where $\delta$ is the strong elastic P-wave $\pi \pi$ phase shifts. (There is an ambiguity of \pm sign on the RHS of Eq. (6); the choice of the sign + was made is due to the normalisation $V(0)=1$ and the phenomenology, e.g. the rms pion radius is positive). Because the real and imaginary parts are related by dispersion relation, it is important to know accurately $\operatorname{Im} V(z)$ over a large energy region. Below $1.3 \mathrm{GeV}, \operatorname{ImV}(z)$ can be determined accurately because the modulus of the vector form factor [11,12] and the corresponding P-wave $\pi \pi$ phase shifts are well measured [13-15].

Using the experimental data on the pion form factor and the corresponding $\pi \pi$ phase shifts, $\operatorname{Im} V(z)$ with experimental errors is given in Fig. 1. Similarly in Fig. 2, $\operatorname{ReV}(s)$ with experimental errors is given using the expression $\operatorname{ReV}(s)=|V(s)| \cos \delta$.

One first shows how the coefficients of the Taylor's series around $s=0$ can be evaluated in terms of $\operatorname{ImV}(s)$ then later, the experimental $\operatorname{ImV}(s)$ and $\operatorname{ReV}(s)$ will be used to test the validity of various theoretical model calculations.

Following the usual definition, $\left.V(s)=1+\frac{1}{6}<r_{V}^{2}\right\rangle s+c s^{2}+d s^{3}+\ldots$ one has $\left\langle r_{V}^{2}\right\rangle=$ $6 a_{1}, c=a_{2}, d=a_{3}$ etc. If one makes a weak assumption that $V(s) / s \rightarrow 0$ as $s \rightarrow \infty$, using Eq. (5) one gets the following results:

$$
<r_{V}^{2}>=0.44 \pm 0.015 \mathrm{fm}^{2} ; c=3.90 \pm 0.10 \mathrm{GeV}^{-4} ; d=9.70 \pm 0.40 \mathrm{GeV}^{-6}
$$

where the upper limit of the integration is taken to be $1.7 \mathrm{GeV}^{2}$. From the $2 \pi$ threshold to $0.5 \mathrm{GeV}$ the experimental data on the the phase shifts are either poor or unavailable, an extrapolation procedure has to be used. For this purpose the results of models 1 and 2, to be discussed later, were used for $\operatorname{ImV}(s)$. They contribute, rspectively, 5\%,15\% and 30\% to the $a_{1}, a_{2}$ and $a_{3}$ sum rules.

Because of the assumed high energy behavior, one cannot calculate here the pion charge $a_{0}$. From these results the radius of convergence for the Taylor's series is (much) less than $1 G e V^{2}$. In fact, a reasonable approximation for this series is the Taylor's series expansion of $V(s) \simeq 1 /(1-$ $a_{1} s$ ). This result is not surprising because it is the zero width approximation for Eq. (3). Away from the $\rho$ resonance, it is a better approximation than the ChPT calculations for the vector pion form factor.

If one was willing to make a stronger assumption that the form factor vanished asymptotically, then one would be able to calculate the pion charge $a_{0}$ and of course also higher derivatives. A straightforward calculation gives $a_{0}=1.02 \pm .08$, where the upper limit of the integration is taken to be $1.7 \mathrm{GeV}^{2}$. The Ward identity requires $a_{0}$ to be exactly unity. This calculation is to illustrate the fact that the $\rho$ resonance gives the major contribution and almost saturates the sum 


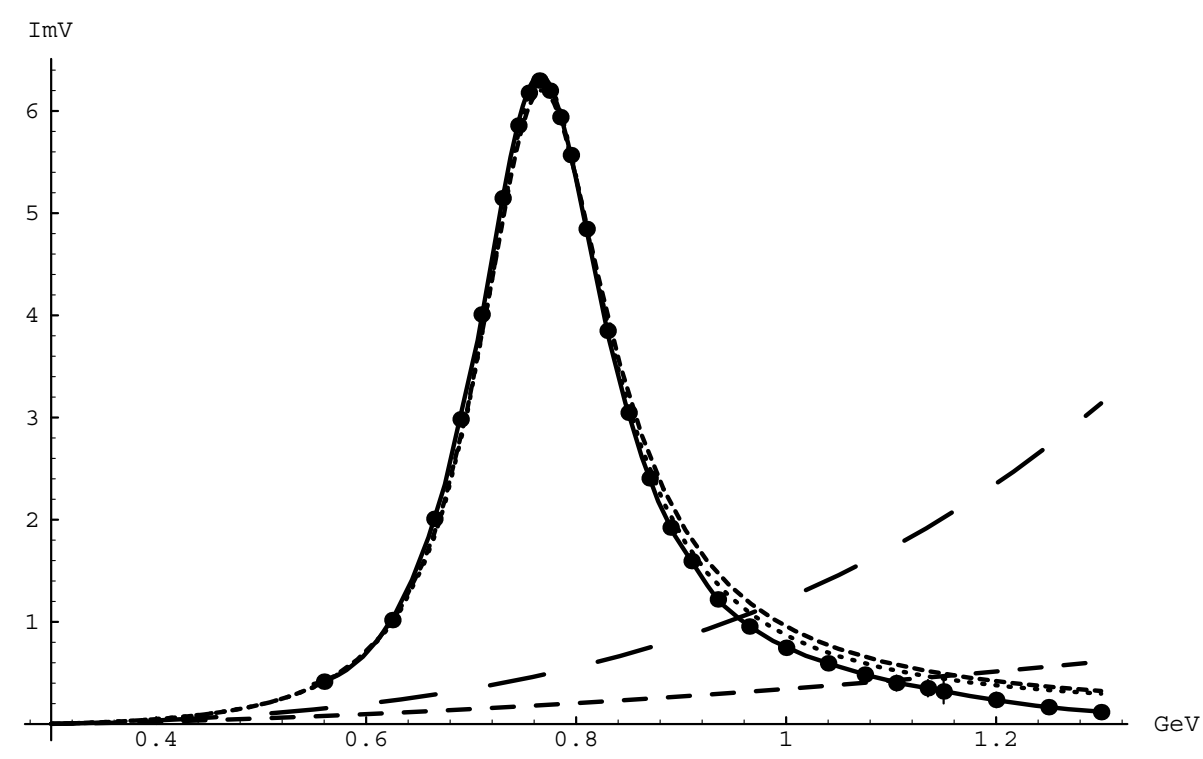

Fig. 1. The imaginary part of the vector pion form factor $\operatorname{Im} V(s)$, given by Eq. (6), as a function of the energy in the $\mathrm{GeV}$ unit. The experimental data of the modulus of the form factor and the P-wave $\pi \pi$ phase shifts are taken from the refences [11-15]. The solid curve is the the experimental results with experimental errors; the long-dashed curve is the two-loop ChPT calculation, the medium long-dashed curve is the one-loop ChPT calculation, the short-dashed curve is from the modified unitarized one-loop ChPT calculation, Eq. (8), and the dotted curve is the unitarized two-loop calculation of Hannah [19].

rule for the charge; it will not be used in the following. The higher derivative sum rules are much less sensitive to the high energy behavior of $\operatorname{ImV}(z)$ because of the corresponding weight factor.

It is possible to estimate the high energy contribution to the sum rules by fitting the asymptotic value of the pion form factor by the expression, $V(s)=-\frac{0.25}{s} \ln \left(-s / s_{\rho}\right)$ which fits equally well the large energy behavior of the time-like and space-like pion form factor, then the integration of the sum rule from $1.7 \mathrm{GeV}^{2}$ to infinity can be estimated. The r.m.s. radius is then increased by $2 \%$ and is completely negligible for the values of $c$ and $d$. (The additional contribution to the $a_{0}$ sum rule is $15 \%$ ).

Notice that the determination of the pion r.m.s. agrees well with its direct experimental value of $0.439 \pm .008 \mathrm{fm}^{2}[16]$ and with comparable errors. The determination of the values of $c$ given by emperical fits to the data using the vector meson dominance models, quoted as having a large error of $30 \%$, is due to the use of models having different values for the form factor at the $\rho$ mass [5,12]. The present determination of $c$ as well as of $d$ has an error of only a few percents.

It is now possible to compare these experimental results with existing calculations.

The first model is obtained using the inverse amplitude method for the pion form factor at one-loop level. It can also be obtained by using the $(0,1)$ Padé approximant method for the oneloop ChPT calculation. The result is well known: by fitting the time like the pion form factor using the experimental $\rho$ mass, the r.m.s. radius is $10 \%$ too small. The calculated $\rho$ width is $0.156 \mathrm{GeV}$ which is satisfactory but the maximum value of square of the modulus of the pion form factor is 


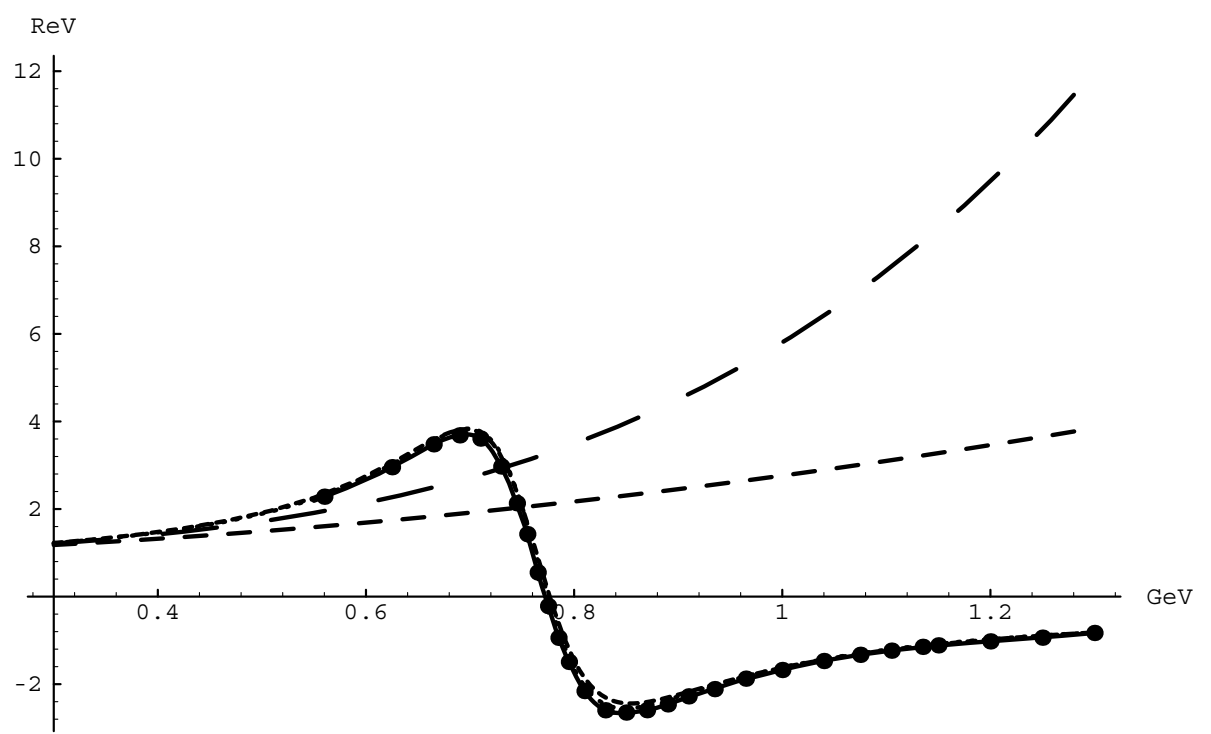

Fig. 2. The real parts of the pion form factor as a function of energy. The curves are as in Fig. 1.

only 31 which is too low compared with the experimental data value of $40.5 \pm 0.6$ as given directly by the $\tau$ decay data.

This result is based on the assumption of the elastic unitarity which should be correct for an energy below $1.3 \mathrm{GeV}$ but is certainly incorrect above this energy. One should phenomenologically correct for this strong assumption. To do this one can simulate the inelastic effect, the contribution of higher resonances of the same quantum number as the vector meson $\rho$ by the presence of the polynomial ambiguity in the phase representation of the form factor i.e. a zero in the form factor. Instead of Eq. (3), the time-like pion form factor data can now be fitted by $[17,18]$ :

$$
V(s)=\frac{1+\alpha s / s_{\rho}}{1-s / s_{R}-\frac{1}{96 \pi^{2} f_{\pi}^{2}}\left\{\left(s-4 m_{\pi}^{2}\right) H_{\pi \pi}(s)+2 s / 3\right\}}
$$

where $f_{\pi}=0.093 \mathrm{GeV}$, and $s_{R}$ is related to the $\rho$ mass squared $s_{\rho}$ by requiring that the real part of the denominator of Eq. (8) vanishes at the $\rho$ mass.

The experimental data can be fitted with a $\rho$ mass equal to $0.773 \mathrm{GeV}$ and $\alpha=0.14$. This is a two parameter fit to the experimental data and there are three predictions: the pion r.m.s. radius, the $\rho$ width and the value of the form factor at the $\rho$ mass (or the $\rho$ leptonic width). These results are in excellent agreement with the data $[12,16]$ : the $\rho$ width, defined as the derivative of the phase shift at the $\rho$ mass, is equal to $0.156 \mathrm{GeV}$, the rms radius is predicted to be $0.45 \pm 0.01 \mathrm{fm}^{2}$ and the maximum value of the modulus squared of the pion form factor at the $\rho$ resonance is 39.2.

The second model, which is more complicated, but is more complete because it is based on the two-loop ChPT calculation with unitarity taken into account. It has the singularity associated with the two loop graphs. By using the same inverse amplitude method as was done with the one-loop amplitude, but by generalizing this method to two-loop calculation, Hannah has recently obtained a remarkable fit to the pion form factor in the time-like and space-like regions. His 
result is equivalent to the $(0,2)$ Padé approximant method as applied to the two-loop ChPT calculation [19]. Unlike Eq. (8) which is a two parameter fit, Hannah calculation is a three parameter calculation which gives the correct maximum value of the pion form factor, the $\rho$ mass and width and the pion r.m.s. radius.

As can be seen from Figs. 1 and 2, the imaginary and real parts of these two models are very much in agreement with the data. A small deviation of $\operatorname{ImV}(s)$ above $0.9 \mathrm{GeV}$ is due to a small deviation of the phases of $V(s)$ in these two models from the data of the P-wave $\pi \pi$ phase shifts. They both give also correct results for $c$ and $d$ as given by the sum rules, Eq.(7). For $0<s<0.4 \mathrm{GeV}^{2}$ the real parts of $V(s)$ of these two models also agree very well with its Taylor's series expansion, using its first 4 terms (not shown).

The results of the imaginary parts of the one and two-loop ChPT calculations are also shown in Fig. 1; it is seen that they differ significantly from the data. At low energy, ChPT results for the real parts are not bad as can be seen from Fig. 2. This is due to the dominance of the real subtraction constants (uncalculable in ChPT scheme) and cannot be used to support the validity of the ChPT as will be discussed below.

At one-loop level, ChPT cannot be used to calculate the rms radius because the loop integral for this quantity is divergent. Using the measured r.m.s. as an input, the one loop ChPT for the pion form factor is given by Eq. (1). The coefficient $c_{1}^{C h P T}$, where the subscript refers to the one-loop level is $c_{1}^{C h P T}=\left(960 \pi^{2} f_{\pi}^{2} m_{\pi}^{2}\right)^{-1} \simeq 0.626 G e V^{-4}$ and $d_{1}^{C h P T}=\left(13440 \pi^{2} f_{\pi}^{2} m_{\pi}^{4}\right)^{-1} \simeq$ $2.30 \mathrm{GeV}^{-6}$ The value for $c$ calculated by the one-loop ChPT is in agreement with a previous determination [19] and is too small by a factor of 7 compared with the sum rule value Eq. (7); $d$ is also a factor of 4 too small compared with the sum rule value, Eq. (7).

At two-loop level [5,6], ChPT cannot be used to calculate the expression for $c$ because of the degree of divergence of the loop integral. One can calculate, however, the expression for $d$. Although an analytical formula can be given, it is simpler to give it numerically: $d_{2}^{C h P T}=$ $4.1 \mathrm{GeV}^{-6}$ which is a factor of 2.5 too small compared with the sum rule value, Eq. (7). The disagreement with the sum rule value is now less than that from the one-loop calculation.

In the following, one follows the standard dispersion relation analysis of the data, i.e. given the imaginary part of an amplitude, one can calculate its real part by dispersion relation. For this purpose, it is important to realize that the equivalent use of the Taylor's series is the dispersion integral (DI) on the R.H.S. of Eq. (4). Because of the use of the elastic unitarity, this analysis is at best valid to a maximum of energy of $0.8-0.9 \mathrm{GeV}$.

One should compare the experimental values of the real part of the DI with those given by models, because they are the direct results of the calculation schemes, unmasked by the dominance of the subtraction constants at low energy. The real parts of the DI can be calculated using $\operatorname{ImV}(s)$ from experimental data, models 1, 2 and ChPT results. In Fig. 3, for clarity, only the two-loop ChPT calculation is plotted. It is seen that the two-loop ChPT results, are too small compared with the corresponding real parts calculated from the data, not only for small s but also for large s (using $n=3$ for the DI). At very low energy one recovers the results of the Taylor's series as discussed above.

The real parts of the DI, calculated using models 1 and 2, are in good agreement with the data at low energy, but show a small deviation from the data above $0.6 \mathrm{GeV}$. This result is expected because models 1 and 2 violate the phase theorem by a small amount above $0.9 \mathrm{GeV}$, as mentioned above. 


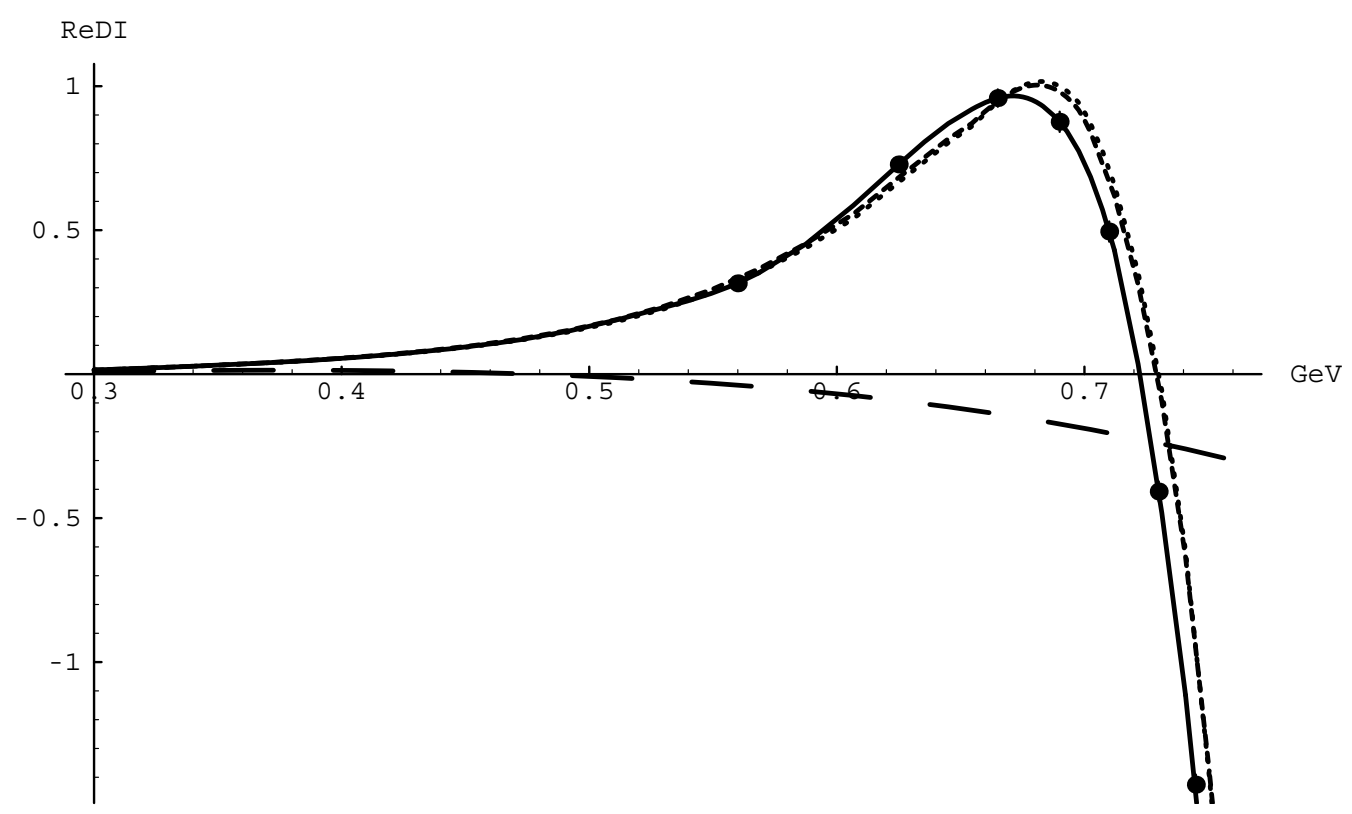

Fig. 3. The real parts of the dispersion integral ReDI as a function of energy (using $n=3$ ) in Eq. (4). The curves are as in Fig. 1; the ChPT one-loop result is not shown.

It is seen that perturbation theory is inadequate for the vector pion form factor calculation for an energy below the $\rho$ mass and even at the point $s=0$ which are fairly far away from the $\rho$ mass. It would be useful to ask how large the hypothetical $\rho$ mass would be in order that perturbation theory could be trusted. It is difficult to answer this question in general but one can take the large $N_{f}$ model [21] as a guide, where $N_{f}$ is the number of flavors. In this model, the explicit solution for the form factor as well as for the scattering amplitude can be given. The expression for the form factor is given by Eq. (3). In order that perturbation theory to be valid, it is required that the coefficient $c$ calculated by the ChPT to be much larger than that given by the large $N_{f}$ model. This condition yields:

$$
s_{R}>>\sqrt{960} f_{\pi} m_{\pi} \simeq 1.26 G e V^{2}
$$

or roughly $s_{\rho}>>1.26 \mathrm{GeV}^{2}$, a condition cannot be satisfied by the physical value of the $\rho$ mass.

The situation may not be as bad for the scalar form factor as can be seen by considering also the large $N_{f}$ model. Instead of Eq. (9) we now have a condition on the scalar r.m.s. of the pion, $1 / 6<r_{\text {scalar }}^{2}>=1 / s_{R}^{\text {scalar }}$ :

$$
s_{R}^{\text {scalar }}>>\sqrt{1920 / 19} \pi f_{\pi} m_{\pi} \simeq 0.41 \mathrm{GeV}^{2}
$$

Using the "experimental" value of the scalar r.m.s. radius, $\left\langle r^{2}\right\rangle=0.6 \mathrm{fm}^{2}[4,5]$, one has $s_{R}^{\text {scalar }} \simeq$ $0.40 \mathrm{GeV}^{2}$ which is equal to the R.H.S. of Eq. (10), instead of being much larger. The situation is better here than the vector case. It might be barely possible to apply the ChPT for the scalar form factor but the accuracy of the perturbative approach could be questionable.

In conclusion, because of the inedequacy of the perturbative loop calculations, one could try two different approaches to this problem. A more radical approach consists in considering 
effective lagrangians just as it was invented for, i.e. low energy theorems; instead of doing perturbative loop calculations, one could try to analytically continue these low energy theorems to the time-like region with the technique of dispersion relation and the constraint of unitarity. A more standard approach is to do perturbative calculation but resumming the perturbative series in order to satisfy unitarity. In both approaches, one could use for example the inverse amplitude, N/D and Padé methods [7,8]. This last method [23], based on the results of the perturbation calculation and briefly reviewed here, could be an useful method but could also give a wrong answer in a number of problems if care was not taken. In general, one does not have a unique prescription of how to handle the strong interaction problem.

\section{ACKNOWLEDGEMENTS}

The author would like to thank Dr. Torben Hannah for a detailed explanation of his calculation of the two-loop vector pion form factor and also for a discussion of the experimental situation on the pion form factor data. Useful conversations with T. N. Pham are acknowleledged.

\section{REFERENCES}

[1] J. F. Donoghue, E. Golowich and B. R. Holstein, Dynamics of the Standard Model, Cambridge Univ. Press, Cambridge, 1992.

[2] S. Weinberg, Physica 96 A (1979) 327.

[3] J. Gasser and H. Leutwyler, Ann. Phys. (N.Y.) 158 (1984) 142.

[4] J. Gasser and H. Leutwyler, Nucl. Phys. B 250 (1985) 465. ibid. B250 (1985) 539.

[5] J. Gasser and Ulf-G Mei $\beta$ ner, Nucl. Phys. B 357 (1991) 90.

[6] G. Colangelo, M. Finkemeier and R. Urech, Phys. Rev. D 54 (1996) 4403.

[7] T. N. Truong, Phys. Rev. Lett. 61 (1988) 2526.

[8] T. N. Truong, Phys. Rev. Lett. 67 (1991) 2260.

[9] K. M. Watson, Phys. Rev. 95 (1954) 228.

[10] F. Guerrero and J. A. Oller, Report No hep-ph/9805334.

[11] L. M. Barkov et al., Nucl. Phys. B 256 (1985) 365.

[12] ALEPH Collaboration, R. Barate et al., Z. Phys. C 76 (1997) 15.

[13] S. D. Protopopescu et al., Phys. Rev. D 7 (1973) 1279.

[14] B. Hyams et al., Nucl. Phys. B 64 (1973) 134.

[15] P. Eastabrooks and A. D. Martin, Nucl. Phys. B 79 (1974) 301.

[16] NA7 Collaboration, S. R. Amendolia et al., Nucl. Phys. B 277 (1986) 168.

[17] Le Viet Dung and Tran N. Truong, Report No hep-ph/9607378.

[18] T. N., Phys. Rev. D 30 (1984) 1509.

[19] T. Hannah, Phys. Rev. D 55 (1997) 5613; Ph. D thesis, Aarhus University (1998).

[20] A. Dobado, M.J. Herrero and T. N. Truong, Phys. Lett. B 235 (1990) 129 - 134.

[21] S. Willenbrock, Phys. Rev. D 43 (1991) 1710 and references cited therein.

[22] A. Dobado and J. R. Pelaez, Phys. Rev. D 56 (1997) 3057.

[23] For other applications of the Padé method see: M.A. Samuel, J. Ellis and M. Karliner, Phys. Rev. Lett. 74 (1995) 4380;

S. J. Brodsky, J. Ellis, E. Gardi, M. Karliner, M. A. Samuel, Phys. Rev. D 56 (1997) 6980. 